\title{
Programmed intermittent epidural bolus versus continuous epidural infusion for postoperative analgesia after major abdominal and gynecological cancer surgery: a randomized, triple-blinded clinical trial
}

Thomas Wiesmann* (D), Lilli Hoff, Lara Prien, Alexander Torossian, Leopold Eberhart, Hinnerk Wulf and Carsten Feldmann

\begin{abstract}
Background: Continuous epidural infusion (CEI) is the standard application setting for epidural infusion. A new mode, the programmed intermittent epidural bolus (PIEB) technique, showed reduced local anesthetic (LA) consumption and improved analgesia in obstetric analgesia. Goal of this trial was to evaluate the effects of PIEB versus CEl [combined with patient-controlled bolus (PCEA)] on LA consumption and pain scorings in major abdominal cancer surgery.
\end{abstract}

Methods: Following ethical approval, patients scheduled for major abdominal cancer surgery under general anesthesia in combination with epidural analgesia were randomized to receive either a PIEB mode of $6 \mathrm{~mL} / \mathrm{h}$ or a CEI mode set at $6 \mathrm{~mL} / \mathrm{h}$ of ropivacaine $0.2 \%$, both combined with a PCEA mode set at a $4 \mathrm{~mL}$ bolus. LA consumptions and pain scorings were documented until the second postoperative evening.

Results: Eighty-four datasets were analyzed (CEI: $n=40$, PIEB: $n=44$ ). Regarding the primary endpoint, cumulative LA PCEA bolus volumes until day 2 differed significantly between the groups [PIEB $10 \mathrm{~mL}(2-28 \mathrm{~mL}$ ) versus CEI, $28 \mathrm{~mL}$ (12-64 mL), median (25th-75th percentiles), $p=0.002$ ]. Overall, LA consumption volumes were significantly lower in the PIEB group versus in the CEI group [PIEB: $329 \mathrm{~mL}$ (291-341 mL) vs. CEl: $350 \mathrm{~mL}$ (327-381 mL), $p=0.003$ ]. Pain scores were comparable at each time point.

Conclusions: This trial demonstrates reduced needs for PCEA bolus in the PIEB group. There were no clinically relevant benefits regarding morphine consumption, pain scorings, or other secondary outcome parameters.

Trial registration: This study has been registered retrospectively in the ClinicalTrials.gov registry (NCT03378804), date of registration: December, 20th 2017.

\footnotetext{
* Correspondence: wiesmann@med.uni-marburg.de

Department of Anesthesiology and Intensive Care Medicine, Philipps

University Marburg, University Hospital Marburg (UKGM - Campus Marburg),

Baldinger Strasse, 35033 Marburg, Germany
}

(c) The Author(s). 2018 Open Access This article is distributed under the terms of the Creative Commons Attribution 4.0 International License (http://creativecommons.org/licenses/by/4.0/), which permits unrestricted use, distribution, and reproduction in any medium, provided you give appropriate credit to the original author(s) and the source, provide a link to the Creative Commons license, and indicate if changes were made. The Creative Commons Public Domain Dedication waiver (http://creativecommons.org/publicdomain/zero/1.0/) applies to the data made available in this article, unless otherwise stated. 


\section{Background}

Major abdominal cancer surgery is regularly linked with intermediate to severe pain following the procedure. Postoperatively, analgesic therapy should be performed by a multimodal combination of systemic analgesics and the use of regional anesthesia techniques such as thoracic epidural analgesia [1, 2]. Additional proven effects of epidural analgesia such as reduced time to the recovery of bowel function are beneficial after abdominal surgery [3]. Epidural infusion is usually administered by an elastomeric or electronic pump delivering continuous local anesthetic (LA) flow via the indwelling epidural catheter, in addition to patient-controlled epidural bolus (PCEA) applications. Recent advances in infusion technology have resulted in the technique of a programmed intermittent epidural bolus (PIEB) at a set interval [4]. In obstetric analgesia, this application mode prompted a significant decrease of LA consumption, [4] potentially due to a better distribution of LA in the epidural space in comparison with the continuous infusion technique. However, this technique has not yet been evaluated for major abdominal surgery.

To our knowledge, this is the first randomized tripleblinded trial (patient blind, researcher blind, blinded statistician) to investigate the effects of PIEB versus continuous epidural infusion (CEI) for postoperative analgesia in combination with PCEA option after major open abdominal and gynecological (i.e., pancreatic, colonic, ovarian, and cervical) cancer surgery on LA consumption (primary endpoint) and postoperative pain management including opioid consumption and potential side effects (secondary endpoints).

\section{Methods}

\section{Ethics, consent \& permissions}

Following approval by the local ethics committee (Ethical Commission, University Hospital Marburg, AZ 119/ 16) and registration in the ClinicalTrials.gov registry (NCT03378804), this triple-blinded, randomized, and controlled single-center study was performed in accordance with the Declaration of Helsinki. Written informed consent was obtained from all participating subjects prior to enrollment. This manuscript adheres to the applicable CONSORT guidelines.

Patients (ASA classification 1 to 4 ) scheduled for elective major abdominal (pancreatic and colonic) and gynecological (ovarian and cervical) cancer surgery with scheduled midline laparotomy were screened between January 2017 and November 2017. Exclusion criteria were age $<18$ years or $>80$ years, an inability to give consent, pregnancy, general contraindications for or an inability to undergo thoracic epidural analgesia techniques, inability to use a patient-controlled epidural analgesia (PCEA) technique, scheduled postoperative mechanical ventilation, and known or suspected allergy to LA.

Predefined dropout criteria were failed epidural anesthesia, revision surgery within the first $24 \mathrm{~h}$ and mechanical ventilation during intensive care unit stay.

\section{Anesthesia, surgery, and postoperative analgesia}

In the induction area, standard monitoring was applied according to current national guidelines. Insertion of a thoracic epidural catheter (Perifix; B. Braun, Melsungen, Germany), using a loss-of-resistance technique was performed after skin disinfection in the sitting position at the Th8-Th9 or the Th9-Th10 interspaces under mild sedation using $5 \mu \mathrm{g}$ to $15 \mu \mathrm{g}$ of intravenous (IV) sufentanil. Following negative aspiration and negative response to a test dose of $3 \mathrm{~mL}$ of bupivacaine $0.5 \%$ (without epinephrine), the indwelling catheter was fixed using sterile drapings (Tegaderm; $3 \mathrm{M}$, Maplewood, MN, USA). An initial dose of $15 \mathrm{~mL}$ of ropivacaine $0.375 \%$ was applied via the indwelling epidural catheter with the patient in the supine position. After $15 \mathrm{~min}$, cold/warm sensibility testing was performed bilaterally to evaluate the appropriate spread (multisegmental sensory blockade) of the epidural block.

Anesthesia induction was performed with sufentanil $0.2 \mu \mathrm{g} \mathrm{kg} \mathrm{kg}^{-1}$ to $0.3 \mu \mathrm{g} \mathrm{kg} \mathrm{kg}^{-1}$, propofol $2 \mathrm{mg} \mathrm{kg}^{-1}$ to $3 \mathrm{mg} \mathrm{kg}-1$, and cisatracurium $0.1 \mathrm{mg} \mathrm{kg}^{-1}$ or rocuronium $0.5 \mathrm{mg} \mathrm{kg}^{-1}$ to $0.6 \mathrm{mg} \mathrm{kg}^{-1}$. Intraoperatively, a balanced anesthesia technique using desflurane (target bispectral index values of 35 to 55) and remifentanil $0.1 \mu \mathrm{g} \mathrm{kg}^{-1} \mathrm{~min}^{-1}$ to $0.25 \mu \mathrm{g} \mathrm{kg}{ }^{-1} \mathrm{~min}^{-1}$ was administered. Intraoperatively, all patients underwent active warming to achieve normothermia. All patients received a standardized IV double antiemetic prophylaxis using dexamethasone $4 \mathrm{mg}$ to $8 \mathrm{mg}$ and granisetrone $1 \mathrm{mg}$ or droperidol $0.625 \mathrm{mg}$ to $1.25 \mathrm{mg}$ with regard to patient-specific conditions [5]. Recovery from neuromuscular blockade was monitored in all patients, a reversal was performed individually at the discretion of the respective anesthetist if the Train-of-Four (TOF) ratio did not reach $4 / 4$ and the Double Burst Stimulation (DBS) showed a residual blockade phenomena. Prior to emergence from anesthesia at the end of the operation, all patients received $15 \mathrm{mg} \mathrm{kg}^{-1}$ to $20 \mathrm{mg} \mathrm{kg}^{-1}$ metamizole (dipyrone) as a nonopioid analgesic component of our standard multimodal analgesic regimen.

After surgery, patients were extubated in the operation room and transferred either to the postanesthesia care unit (PACU) or-if so scheduled-directly into the intensive care unit for further care.

\section{Intervention groups}

At the beginning of the operation, a PCEA pump (AmbIT ${ }^{\oplus}$ PIB PCA; Teleflex, Wayne, PA, USA) with a sterile bag 
containing ropivacaine $0.2 \%$ (Naropin $^{\odot} 2 \%$; AstraZeneca, Cambridge, UK) and sufentanil $0.75 \mu \mathrm{g} / \mathrm{mL}^{-1}$ (Sufentanil Hameln, Hameln Pharma, Hameln, Germany) was connected to the epidural catheter. The PCEA pump was either programmed with a CEI of $6 \mathrm{~mL} / \mathrm{h}$ flow or using the programmed intermittent bolus (PIB) mode with $6 \mathrm{~mL}$ bolus every $60 \mathrm{~min}$ according to the randomization result (details are given below). Both pump settings were combined with a patient-controlled bolus option (PCEA) of $4 \mathrm{~mL}$ (lockout time: $30 \mathrm{~min}$ ). Staff members as well as patients were unaware of the randomization results until the time of overall data analysis.

\section{Postoperative analgesia}

Staff nurses and patients were asked to use the PCEA whenever the numeric rating scale (NRS; range: 0-10) for pain at rest of the patient was 4 or higher. Patients were encouraged to use the PCEA during ward stay until postoperative day (POD) 3 whenever they were not receiving adequate pain control (NRS $>4$ ). In the case of insufficient analgesic response to the PCEA bolus (to reach an NRS < 4 within 15-20 min after bolus application, IV rescue analgesia with the opioid piritramide (3.75-7.5 mg intravenously) was allowed as a 'rescue option'. Patients received a combination of oral ibuprofen and metamizole (dipyrone) as part of our institutional multimodal protocol. In patients with repetitive needs for systemic opioids, $10 \mathrm{mg}$ to $20 \mathrm{mg}$ of prolonged-release oxycodone was given orally twice daily.

\section{Sample size calculation}

Sample size calculation was performed according to data on LA consumption differences between CEI and PIB modes in obstetric anesthesia provided by a meta-analysis by George et al. [4] as well as our own historical patient data. Hourly LA consumption was calculated to equal $5 \mathrm{~mL} / \mathrm{h}$ of ropivacaine $0.2 \%$ [standard deviation (SD): $1.7 \mathrm{~mL} / \mathrm{h}$ ] in the conventional group in contrast to the programmed intermittent epidural group, which was calculated to equal $4 \mathrm{~mL} / \mathrm{h}$ ropivacaine $0.2 \%$ (SD: $1.35 \mathrm{~mL} / \mathrm{h})$. Given an alpha value of 0.05 and a beta value of 0.8 , we calculated the minimum required sample size of patients per group to be 37 to detect a group difference of $1 \mathrm{~mL} / \mathrm{h}$ assuming a standard deviation in the two groups of one third of their means (PASS 15; NCSS, Kaysville, UT, USA). This number was initially increased to 45 patients per group to compensate for dropouts. During the active study period, a relevant number of dropouts and serious protocol violations (which were not related to the study, e.g. reoperations) occurred (Fig. 1). Thus, after obtaining permission from the ethical commission, another 20 participants were recruited and randomized to achieve the relevant number of included patients for statistical analysis as determined by the sample size calculation. Hence, overall 110 patients were finally randomized.

\section{Randomization, allocation, and blinding}

A computer-generated sequence of random numbers was used to randomize the study participants with a 1:1 ratio of CEI to PIEB, both in combination with a PCB mode, using the specific modes of the PCEA pump system. To avoid imbalances due to the three different types of abdominal surgery (colonic/pancreatic/gynecological surgeries), a stratified randomization approach was used. The randomization sequence was generated by an investigator (LE) who had no further involvement in the patient treatment using the aforementioned Internet-based randomization tool (www.random.org) to generate a block randomized list (block size of 6 patients). The allocation was concealed in sealed, opaque, sequentially numbered envelopes. For each study participant, one envelope was handed to an assistant not involved with the study or a patient care assistant who was assigned to program the electronic pump for the epidural analgesia according to the randomization result. In a running pump LCD display, the overall amount of applied volume is shown but not the respective mode of delivery as programmed by the first, unblended operator. Neither the patients nor the responsible anesthetists or study assistants were aware of the randomization results. According to the triple-blinded study approach, unblinding was performed following completed statistical analysis of the blinded study allocation.

\section{Primary and secondary endpoints}

The primary endpoint of this trial was cumulative overall LA volumes of the PCB mode until $48 \mathrm{~h}$ after surgery. Secondary endpoints included delivered CEI or PIEB mode-derived volumes plus delivered PCB volumes (overall LA consumption) as well as the LA volumes per mode (CEI or PIEB) delivered at different time points until the evening of the operation day (POD0) and the first and second postoperative day (POD1-POD2).

Pain was evaluated using the NRS $(0-10$; 0 , no pain; 10 , worst imaginable pain) at rest, during cough, and during movement.

IV morphine equivalents were calculated for the first 3 days separately and overall. Oral and intravenously applied opioids other than IV morphine were converted into IV morphine equivalents (i.e., IV morphine $10 \mathrm{mg}=$ oral morphine $30 \mathrm{mg}=\mathrm{IV}$ hydromorphone $1.5 \mathrm{mg}=$ oral hydromorphone $7.5 \mathrm{mg}=$ oral oxycodone $20 \mathrm{mg}=\mathrm{IV}$ tramadol $100 \mathrm{mg}=\mathrm{IV}$ piritramide $7.5 \mathrm{mg}$ ) according to the published literature [6].

Time to the first passage of flatus and time to the first passage of feces (stool) were calculated as the time of the end of the surgery until the first event of each, respectively. Nausea and vomiting were calculated based 


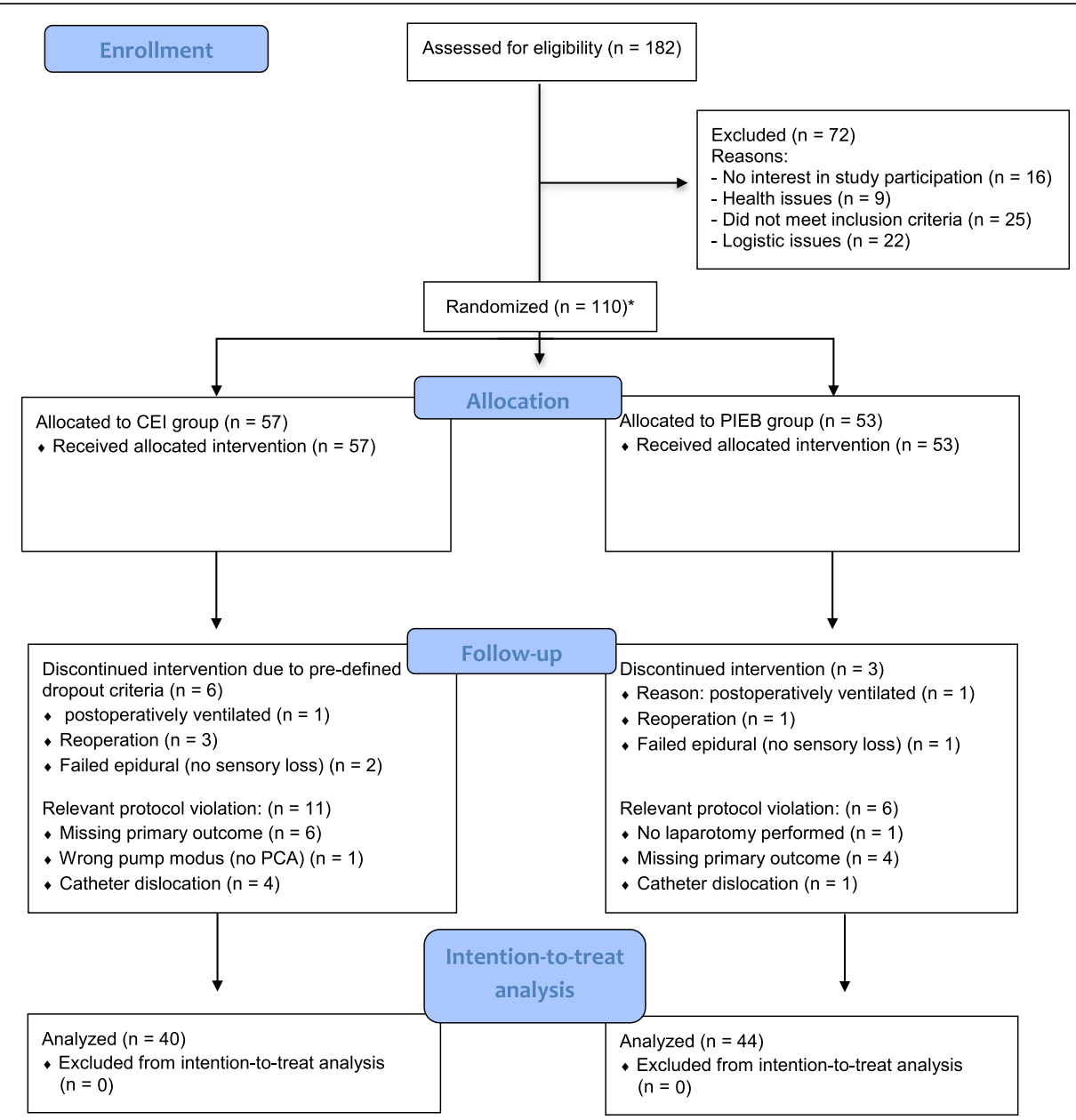

Fig. 1 CONSORT Flowchart. *Initial, randomization of 90 patients was planned. During study period, further ethical permission was given for in total 110 patients due to an unexpected higher rate of drop-outs and protocol violations. For details, see text

on dichotomous variables. Overall patient satisfaction was assessed using a numeric rating score from 0 to 10 .

We assessed sensory spread (by using a standardized cold-warm testing method) bilaterally 15 min after the initial LA bolus application via the indwelling epidural catheter in the preoperative area as well as after surgery at POD0, POD1, and POD2. Patient-reported numbness was investigated as dichotomous variable (yes / no), signs of motor-blockade were investigated according to Bromage's score as an ordinal-scaled variable from 0 to 4 (degrees of motor blockade: 0 , none; 1 , inability to perform hip flexion; 2, inability to perform knee flexion; 3, inability to move legs or feet).

Assessments were performed in a standardized fashion by one of the same two investigators (LH and LP) to reduce interobserver variability.

\section{Statistical analysis}

Continuous variables are presented in the format of the mean \pm SD for normally distributed data or as medians (including the 25th-75th interquartile range) for data with non-normal distributed variables. Nominal and ordinal (categorical) variables are presented as $\mathrm{n}(\%)$. The normality of distribution was tested using histograms and QQ plots as well as statistical analysis using the Shapiro-Wilk test. Modified intention-to-treat statistical analysis was performed for all analyzed parameters. Fisher's exact test for categorical variables or a chi-squared test were applied when appropriate for testing differences in groups. An analysis of continuous variables was performed using the Student's $t$-test for mean differences or the Mann-Whitney $U$ test when nonparametric testing was necessary. Hodges-Lehmann estimators for the location of the shift parameter were given for local anesthetic consumption values for the evaluation of clinically relevant differences. Twosided $p$-values were reported. Statistical significance was judged at a type I error level of 0.05 . Statistical analysis was performed using SPSS (release 22; IBM Corp., Armonk, NY, USA). 


\section{Results}

From January 2017 until November 2017, 182 patients were assessed for eligibility. Of these, 72 patients did not participate in the study (16 patients refused to participate in the study, 25 patients did not meet inclusion criteria, nine patients had medical contraindications, 17 were excluded due to logistic issues such as postponed or cancelled surgery, and five were excluded due to other reasons). Finally, 110 patients were randomized (Fig. 1). Blinded intention-to-treat analysis of 84 patients was performed according to protocol. Details of dropout reasons and relevant protocol violations are given in Fig. 1.

Among the 84 patients analyzed (40 patients in the CEI group and 44 patients in the PIEB group), baseline patient characteristics were found to be similar between the groups (Table 1).

\section{Local anesthetic consumption}

Regarding the primary outcome measurement, the applied PCEA bolus volumes were significantly higher in the CEI group [28 $\mathrm{mL}(12-64 \mathrm{~mL})]$ versus in the PIEB group [10 mL (2-28 ml), $p=0.004$, Table 2]. Additionally, the differences between the need for additional PCEA bolus were statistically different on POD1 and POD2 but not so from the initial time of surgery until the evening of the same day (Table 2).

A statistically significant difference in overall LA consumption was observed in the PIEB group [329 mL (291$341.1 \mathrm{~mL})$; median 25th-75th percentiles] as compared with in the CEI group [350.3 mL (326.5-380.8 mL), $p=$ 0.01 ] until $48 \mathrm{~h}$ postoperatively (POD2). The LA volumes applied by the CEI or PIEB mode alone (without PCEA bolus volumes) were not significantly different between

Table 1 Demographic and perioperative data

\begin{tabular}{|c|c|c|c|}
\hline & Group CEI $(n=40)$ & Group PIEB $(n=44)$ & $p$-value \\
\hline Age, y & $58 \pm 16$ & $63 \pm 12$ & 0.09 \\
\hline Sex, $(M / F)$ & $9 / 31$ & $15 / 29$ & 0.24 \\
\hline Weight, kg & $80 \pm 21$ & $79 \pm 20$ & 0.96 \\
\hline Height, cm & $167 \pm 8$ & $168 \pm 9$ & 0.61 \\
\hline $\mathrm{BMI}, \mathrm{kg} / \mathrm{m}^{2}$ & $29 \pm 7$ & $28 \pm 7$ & 0.81 \\
\hline ASA status $|/||/|||$ & 0/23/17 & $1 / 28 / 15)$ & 0.37 \\
\hline Length of hospital stay, $d$ & $14(10-18)$ & $12(10-16)$ & 0.53 \\
\hline Type of surgery (colonic/pancreatic/gynaecological) & $12 / 09 / 19$ & $14 / 10 / 20$ & 0.84 \\
\hline Length of surgery, min & $205(171-329)$ & 173(135-253) & 0.06 \\
\hline Epidural insertion height (lower spinal segment) & Th10 (Th9-Th11) & Th10 (Th9-Th11) & 0.60 \\
\hline Epidural catheter performance time, min & 12(8-17) & $10(7-12)$ & 0.14 \\
\hline Length of epidural catheter therapy, $d$ & $5(5-7)$ & $5(5-6)$ & 0.33 \\
\hline MAP initial (MAP1), mmHg & $107(91-123)$ & $103(93-114)$ & 0.46 \\
\hline MAP at initial ropivacaine administration (MAP 2), $\mathrm{mmHg}$ & $97(90-116)$ & $100(89-113)$ & 0.81 \\
\hline MAP $15 \mathrm{~min}$ after initial ropivacaine administration (MAP 3), $\mathrm{mmHg}$ & $88(76-97)$ & $86(78-98)$ & 0.94 \\
\hline Sufentanil for EDA placement, $\mu \mathrm{g}$ & $0(0-10)$ & $0(0-5)$ & 0.24 \\
\hline Metamizole intraoperative, $\mathrm{g}$ & $1.5(1-1.5)$ & $1.5(1.5-1.5)$ & 0.76 \\
\hline Metamizole d0, g & $2(1-3.5)$ & $2(1-3)$ & 0.73 \\
\hline Metamizole d1, g & $4(4-4)$ & $4(3-4)$ & 0.22 \\
\hline Metamizole d2, g & $4(4-4)$ & $4(4-4)$ & 0.41 \\
\hline Ibuprofen d0, g & $0.8(0.4-0.8)$ & $0.8(0.6-0.8)$ & 0.66 \\
\hline Ibuprofen d1, g & $1.2(0.8-1.4)$ & $1.2(0.6-1.2)$ & 0.83 \\
\hline Ibuprofen $\mathrm{d} 2, \mathrm{~g}$ & $1.2(1.2-1.6)$ & $1.2(1.2-1.8)$ & 0.33 \\
\hline Opioids d0, \% (yes/no) & $30(12 / 28)$ & $36.4(16 / 28)$ & 0.54 \\
\hline Opioids d1, \% (yes/no) & $45(18 / 22)$ & $40.1(18 / 26)$ & 0.71 \\
\hline Opioids d2, \% (yes/no) & $67.5(27 / 13)$ & $50(22 / 22)$ & 0.11 \\
\hline Opiods d0-2, \% (yes/no) & $75(30 / 10)$ & $66(29 / 15)$ & 0.37 \\
\hline
\end{tabular}

Values are expressed as the mean \pm SD, median (25th-75th percentile), number of patients $(\mathrm{n})$, or absolute numbers (\%). PIEB programmed intermittent epidural bolus group, CEI continuous epidural infusion group, BMI body mass index, ASA American Society of Anesthesiology, EDA epidural anesthesia, MAP mean arterial pressure, $L A$ local anaesthetic; Statistical significance was tested using Mann-Whitney-U-Testing, T-Testing or Chi-square where appropriate, two-sided $p$-value. Level of significance $p<0.05$ 
Table 2 Local anesthetic consumption

\begin{tabular}{lllll}
\hline & Group CEl $(n=40)$ & Group PIEB $(n=44)$ & $p$-value & HL-Estimator $(95 \%$ Cl) \\
\hline LA PCA Bolus d0-d2, ml & $28(12-64)$ & $10(2-28)$ & $0.002^{*}$ & $16(4 ; 28)$ \\
LA PIEB/CEl amount d0-d2, ml & $323.5(286.6-336.7)$ & $311.4(263-330.2)$ & 0.161 & $10.2(-4 ; 30.6)$ \\
Overall LA consumption d0-d2, ml & $350.3(326.5-380.8)$ & $329(291-341.1)$ & 0.003 & $29.55(10.7 ; 52.1)$ \\
LA PCA Bolus d0, ml & $0(0-8)$ & $0(0-4)$ & 0.046 & $0(0 ; 0)$ \\
LA PCA Bolus d1, ml & $18(4-26)$ & $4(0-14)$ & $0.002^{*}$ & $8(4 ; 16)$ \\
LA PCA Bolus d2, ml & $12(4-34)$ & $4(0-12)$ & $0.010^{*}$ & $4(0 ; 12)$ \\
LA PIEB/CEl amount d0, ml & $50.9(36-55.7)$ & $48(36-54)$ & 0.238 & $2.9(-1,8 ; 6.8)$ \\
LA PIEB/CEl amount d1, ml & $140.9(132.4-145.2)$ & $132.3(120-141.7)$ & $0.015^{*}$ & $8.3(1.2 ; 17.2)$ \\
LA PIEB/CEl amount d2, ml & $135.2(110.6-141.4)$ & $131(109.5-140.1)$ & 0.588 & $2.3(-6.6 ; 11)$
\end{tabular}

$L A$ local anesthestic, $d 0$ day of operation; $d 1 / d 2$ first/second postoperative day; PIEB programmed intermittent epidural bolus, $C E I$ continuous epidural infusion, PCA patient controlled bolus, HL Hodges-Lehman. Values are expressed as the median (25th-75th percentiles), Mann-Whitney-U-Testing, two-sided p-value. Uncorrected $p$-values are displayed. Level of significance $p<0.05 .{ }^{*}$, significant

the CEI group [323.5 $\mathrm{mL}(286.6-336.7 \mathrm{~mL})]$ and the PIEB group [311.4 $\mathrm{mL}(263-330.2 \mathrm{~mL}), p=0.161]$.

\section{Pain scorings and analgesic therapy}

NRS value at rest, during cough, and during movement did not differ significantly between the CEI and PIEB groups for each given time point (Table 3). The amounts of ibuprofen and metamizole given as part of our multimodal regimen also did not differ between the groups for the given time points (Table 1). Additionally, overall, the need for opioids (as calculated as IV morphine equivalents) was not significantly different in the CEI group [15 mg [2.5-22.5 mg]] versus in the PIEB group [7.5 $\mathrm{mg}(0-20 \mathrm{mg}), p=0.27$ ] between the end time of the surgery and POD2.

\section{Side effects}

Patients in both groups had low needs of necessary norepinephrine therapy in the evening after surgery (3/46 in the CEI group vs. $3 / 44$ in the PIEB group, Table 4$)$. On POD1, one patient in the CEI group and two patients in the PIEB group, respectively, required norepinephrine, whereas, on POD2, no patients required additional norepinephrine.

Table 3 Pain scoring

\begin{tabular}{|c|c|c|c|}
\hline & Group CEI $(n=40)$ & Group PIEB $(n=44)$ & $p$-value \\
\hline NRS rest $\mathrm{d} 0$ & $0(0-0)$ & $0(0-0)$ & 0.21 \\
\hline NRS rest $\mathrm{d} 1$ & $0(0-3)$ & $0(0-2.8)$ & 0.94 \\
\hline NRS rest $\mathrm{d} 2$ & $0.5(0-3)$ & $0(0-2.5)$ & 0.34 \\
\hline NRS cough do & $0(0-3)$ & $0(0-0)$ & 0.06 \\
\hline NRS cough d1 & $3(1-6)$ & $3(0-5)$ & 0.49 \\
\hline NRS cough $d 2$ & $2.5(0.5-5.5)$ & $2.5(0-6)$ & 0.81 \\
\hline NRS movement do & $0(0-3)$ & $0(0-0)$ & 0.32 \\
\hline NRS movement $\mathrm{d} 1$ & $3(1-5.5)$ & $3(0-4.5)$ & 0.39 \\
\hline NRS movement $\mathrm{d} 2$ & $3(0-5)$ & $2(0-4.5)$ & 0.41 \\
\hline Morphine IV d0, mg & $5(5-7.5)$ & $5(5-5)$ & 0.03 \\
\hline Morphine IV d1, mg & $10(10-10)$ & $10(10-10)$ & 0.53 \\
\hline Morphine IV d2, mg & $10(10-15)$ & $10(10-10)$ & 0.94 \\
\hline Morphine IV d0-d2, mg & $15(2.5-22.5)$ & $7.5(0-20)$ & 0.27 \\
\hline Opioid need d0, yes / no & $12 / 28$ & $16 / 28$ & 0.54 \\
\hline Opioid need d1, yes / no & $18 / 22$ & $18 / 26$ & 0.71 \\
\hline Opioid need d2, yes / no & $27 / 13$ & $22 / 22$ & 0.11 \\
\hline Opioid need d0-d2, yes / no & $30 / 10$ & $29 / 15$ & 0.37 \\
\hline
\end{tabular}

Values are expressed as the median (25th-75th percentile and as two-sided p-value of the Mann-Whitney -U test. Uncorrected $p$-values are displayed. Level of significance $p<0.05$. No significant differences were found after Bonferroni correction. NRS numeric rating scale, d0-3 postoperative day $0-3, L A$ local anaesthetic 
Table 4 Side effects / Clinical parameters

\begin{tabular}{|c|c|c|c|}
\hline & Group CEI $(n=40)$ & Group PIEB $(n=44)$ & $p$-value \\
\hline Vasopressor need d0, \% (yes/no) & $7.5(3 / 37)$ & $6.8(3 / 41)$ & 0.88 \\
\hline Vasopressor need d1, \% (yes/no) & $2.5(1 / 39)$ & $4.5(2 / 42)$ & 0.62 \\
\hline Vasopressor need d2, \% (yes/no) & $0(0 / 40)$ & $0(0 / 44)$ & 1.00 \\
\hline Nausea d0, \% (yes/no) & $20(8 / 32)$ & $18.2(8 / 36)$ & 0.79 \\
\hline Nausea d1, \% (yes/no) & $22.5(9 / 31)$ & $25(11 / 33)$ & 0.79 \\
\hline Nausea d2, \% (yes/no) & $32.5(13 / 27)$ & $13.6(6 / 38)$ & 0.04 \\
\hline Vomiting d0, \% (yes/no) & $17.5(7 / 33)$ & $11.4(5 / 39)$ & 0.40 \\
\hline Vomiting d1, \% (yes/no) & $20(8 / 32)$ & $22.7(10 / 34)$ & 0.76 \\
\hline Vomiting d2, \% (yes/no) & $17.5(7 / 33)$ & $15.9(7 / 37)$ & 0.85 \\
\hline Satisfaction d0, NRS & $10(10-10)$ & $10(10-10)$ & 0.15 \\
\hline Satisfaction d1, NRS & $10(10-10)$ & $10(9-10)$ & 0.19 \\
\hline Satisfaction d2, NRS & $10(8-10)$ & $10(8-10)$ & 0.84 \\
\hline EDA catheter removal, day & $5(5-7)$ & $5(5-6)$ & 0.33 \\
\hline Time to first flatus, hours & $22.8(18.6-43.8)$ & $23.1(20.3-45.5)$ & 0.25 \\
\hline Time to first defecation, hours & $71.2(40.8-119)$ & 65.8(32.4-93.5) & 0.54 \\
\hline
\end{tabular}

Values are expressed as the median (25th-75th percentiles) or numbers (percents) and as two-sided p-value of the Mann-Whitney -U test or Fisher's exact test when appropriate. Uncorrected $p$-values are displayed. Level of significance $p<0.05$. NRS numeric rating scale, $d 0-3$ postoperative day $0-3$, LA local anaesthetic, $E D A$ epidural anesthesia

Time to first passage of flatus was not significantly different in the CEI group [22.8 $\mathrm{h}(17.6-42.2 \mathrm{~h})]$ as compared with in the PIEB group [23.1 h (20.3-45.5 h), $p=0.25$ ]. The time to first passage of feces after surgery was also not significantly different between the CEI group [71.2 $\mathrm{h}(40.8-119 \mathrm{~h})]$ and the PIEB group [65.8 $\mathrm{h}$ $(32.4-93.5 \mathrm{~h}), p=0.54]$. Frequencies of nausea and vomiting were not significantly different between groups at the evening of the operation, the first and the second postoperative day. Patient satisfaction at $\mathrm{d} 1$ and $\mathrm{d} 2$ was not significantly different between groups (Table 4). Additional information regarding sensory spread and motor blockade testing is given in the Additional file 1 .

\section{Discussion}

To our knowledge, this is the first randomized trial that has investigated different modes of epidural local anesthetic application (continuous vs. programmed intermittent technique) in patients undergoing major abdominal cancer surgery. Our study shows a statistically significant decrease of administered ropivacaine $0.2 \%$ until POD2 in patients who experienced PIEB as compared with CEI, both in combination with a patient controlled bolus (PCB) option. Overall pain scores did not differ significantly between the groups at rest, during cough, or during mobilization for each given time point until POD2. Additionally, overall postoperative morphine consumption showed comparable low values. Time to first passage of flatus or feces (stool) were not significantly different between the groups.

\section{Analgesic effects and morphine consumption}

Our study results are consistent with previously published obstetric data showing reduced local anesthetic consumption when using a PIEB technique versus a traditional CEI [4, 7-10]. However, the overall reduction of LA consumption between the day of operation and POD2 was approximately $15 \mathrm{~mL}$, resulting in questionable clinical relevance. Again, this is in line with previous reports that showed only mild reductions in LA volumes when the PIEB technique was used. On the other hand, this significant difference is explainable by a reduction of applied PCEA bolus volume as a surrogate of better analgesia. This effect of reduced PCEA bolus consumption in the PIEB group as compared with in the CEI group was consistently shown on POD1 and POD2 but not on the day of the surgery. This may be the result of the large initial bolus of $15 \mathrm{~mL}$ of ropivacaine $0.375 \%$ that was used for improved sensory testing quality of the epidural technique in the induction area prior to surgery. Pain scores were comparable between the groups, which is explainable by the fact that IV opioids were given as a rescue option in addition to the patient controlled bolus application option of the epidural infusion pump.

\section{Limitations}

Our study has several limitations and strengths that need to be addressed.

First, there was a relevant number of dropouts and protocol violations (26 of 110 randomized patients). This was the result of several relevant factors: specifically, reoperations due to early complications as well as unplanned 
prolonged mechanical ventilation with necessary sedation were classified as severe protocol violations as they potentially interfered with the primary and secondary endpoints of our study. Thus, we decided to exclude patients who experienced these scenarios from our analysis. Additionally, the decision to choose the local anesthetic consumptions as endpoints were problematic in our clinical practice, as some staff members accidentally switched empty LA bags of our study patients and cleared the pump history, which resulted in the loss of the endpoint parameters in several patients.

As a second limitation, the decision to choose local anesthetic consumption as a primary endpoint might be questioned by some colleagues. This surrogate endpoint was selected for use in our study as previous investigations (mainly in obstetric populations) showed no differences in pain scores or rescue opioid use between CEI and PIEB modes. Moreover, from an ethical point of view, it would be inhuman to expose one trial group to higher pain scores by denying rescue opioids just to aim for a difference in pain scorings. Even a difference of $10 \mathrm{~mm}$ in the $100 \mathrm{~mm}$ visual analogue scale for pain was noted by patients as a relevant difference in pain according to the results of a recent study by Myles et al [11].

Lastly, the optimal PIEB mode has yet to be determined with regard to programmed bolus volume, time interval, local anesthetic, or its concentration $[8,12,13]$. Thus, we chose a pragmatic approach using our standard settings for epidural catheters of $6 \mathrm{~mL} / \mathrm{h}^{-1}$ for the CEI group and the same volume for the PIEB in the PIEB group. We did not perform an economic analysis of the different types of epidural infusion. PIEB modes are restricted to specific types of commercially available electronic pumps, which potentially might result in higher costs in comparison with other pump systems. Moreover, the programmed intermittent bolus application is applied by the pumps with a higher pressure than in continuous infusion technique, resulting in higher energy consumption as compared with the continuous infusion [14]. When using alkaline batteries, a shorter interval of battery exchange might have a considerable economic and ecologic impact [15]. Thus, if available, A/C adapters or rechargeable batteries should be considered when using PIEB modes, regardless of the specific manufacturer.

\section{Conclusion}

In this triple-blinded randomized trial, local anesthetic consumption was significantly lower in patients with a PIEB versus a standard CEI. However, overall pain scores were comparable, as were side effects and patient satisfaction scorings. From a clinical perspective, the overall reduction of local anesthetic consumption was mild comparing the PIEB mode with the CEI mode. Hence, the use of PIEB mode in thoracic epidural analgesia after major abdominal surgery should be further evaluated with regard to optimized programmed bolus volumes and time intervals and outcome before widespread implementation in the clinical setting.

\section{Additional file}

Additional file 1: Sensory \& motor blockade testing. (DOCX 104 kb)

\section{Abbreviations}

ASA: American Society of Anesthesiology; CEl: Continuous epidural application; d: Day; EDA: Epidural anesthesia; LA: Local anesthetics; MAP: Mean arterial pressure; ml: Millilitres; NRS: Numeric rating scale; PACU: Postoperative care unit; PCB: Patient controlled bolus; PCEA: Patient controlled epidural analgesia; PIEB: Programmed intermittent epidural bolus; POD: Postoperative day; SD: Standard deviation

\section{Acknowledgements}

We thank our staff team in the OR, PACU and the acute pain service for their efforts in performing this study.

\section{Funding}

This study was performed with institutional funding

PCEA pumps were provided by Teleflex Germany (Kernen, Germany).

\section{Availability of data and materials}

The full study protocol and raw data set can be obtained from Dr. Thomas Wiesmann (wiesmann@med.uni-marburg.de).

\section{Authors' contributions}

TW: study design, data analysis, interpretation, manuscript draft; LH: data collection \& analysis, interpretation, manuscript draft; LP: data collection \& interpretation, manuscript draft; AT, study design, data analysis, manuscript draft; LE: study design, data analysis \& interpretation; HW: study design, data interpretation, manuscript draft; CF: study design, data collection \& interpretation, manuscript draft; All authors read and approved the final manuscript.

Ethics approval and consent to participate

Ethics approval was obtained by the local ethics committee (Ethical Commission, University Hospital Marburg, AZ 119/16) This study was performed in accordance with the Declaration of Helsinki. Written informed consent was obtained from all participating subjects prior to enrollment.

\section{Consent for publication}

Not applicable

\section{Competing interests}

Thomas Wiesmann has received speaker fees from B. Braun. Hinnerk Wulf has received speaker fees from Teleflex Medical, Vygon, B Braun, Pajunk, and Sintetica.

Leopold Eberhart has received honoraria for consulting and speaker fee from Baxter $\mathrm{GmbH}$, Fresenius $\mathrm{GmbH}$, Grunenthal $\mathrm{GmbH}$, and TEVA ratiopharm $\mathrm{GmbH}$.

All other authors have no competing interest to disclose.

\section{Publisher's Note}

Springer Nature remains neutral with regard to jurisdictional claims in published maps and institutional affiliations.

Received: 17 July 2018 Accepted: 9 October 2018

Published online: 30 October 2018

\section{References}

1. Feldheiser A, Aziz O, Baldini G, Cox BPBW, Fearon KCH, Feldman LS, et al. Enhanced recovery after surgery (ERAS) for gastrointestinal surgery, part 2: consensus statement for anaesthesia practice. Acta Anaesthesiol Scand. 2016;60:289-334. 
2. Guay J, Nishimori M, Kopp SL. Epidural local anesthetics versus opioid-based analgesic regimens for postoperative gastrointestinal paralysis, vomiting, and pain after abdominal surgery: a Cochrane review. Anesth Analg. 2016; 123:1591-602.

3. Pöpping DM, Elia N, Van Aken HK, Marret E, Schug SA, Kranke P, et al. Impact of epidural analgesia on mortality and morbidity after surgery: systematic review and meta-analysis of randomized controlled trials. Ann Surg. 2014;259:1056-67.

4. George RB, Allen TK, Habib AS. Intermittent epidural bolus compared with continuous epidural infusions for labor analgesia: a systematic review and meta-analysis. Anesth Analg. 2013;116:133-44.

5. Wiesmann T, Kranke P, Eberhart L. Postoperative nausea and vomiting - a narrative review of pathophysiology, pharmacotherapy and clinical management strategies. Expert Opin Pharmacother. 2015;16(7):1069-770.

6. Berdine HJ, Nesbit SA. Equianalgesic dosing of opioids. J Pain Palliat Care Pharmacother. 2006;20:79-84.

7. Nunes J, Nunes S, Veiga M, Cortez M, Seifert I. A prospective, randomized, blinded-endpoint, controlled study - continuous epidural infusion versus programmed intermittent epidural bolus in labor analgesia. Braz J Anesthesiol. 2016;66:439-44.

8. Wong CA, Ratliff JT, Sullivan JT, Scavone BM, Toledo P, McCarthy RJ. A randomized comparison of programmed intermittent epidural bolus with continuous epidural infusion for labor analgesia. Anesth Analg. 2006;102:904-9.

9. Capogna G, Camorcia M, Stirparo S, Farcomeni A. Programmed intermittent epidural bolus versus continuous epidural infusion for labor analgesia: the effects on maternal motor function and labor outcome. A randomized double-blind study in nulliparous women. Anesth Analg. 2011;113:826-31.

10. McKenzie CP, Cobb B, Riley ET, Carvalho B. Programmed intermittent epidural boluses for maintenance of labor analgesia: an impact study. Int J Obstet Anesth. 2016;26:32-8.

11. Myles PS, Myles DB, Galagher W, Boyd D, Chew C, MacDonald N, et al. Measuring acute postoperative pain using the visual analog scale: the minimal clinically important difference and patient acceptable symptom state. Br J Anaesth. 2017;1 18:424-9.

12. Zakus P, Arzola C, Bittencourt R, Downey K, Ye XY, Carvalho JC. Determination of the optimal programmed intermittent epidural bolus volume of bupivacaine $0.0625 \%$ with fentanyl $2 \mu \mathrm{gg} . \mathrm{Ml}$-1at a fixed interval of forty minutes: a biased coin up-and-down sequential allocation trial. Anaesthesia. 2017:116:133-7.

13. Carvalho B, George RB, Cobb B, McKenzie C, Riley ET. Implementation of programmed intermittent epidural bolus for the maintenance of labor analgesia. Anesth Analg. 2016;123:965-71

14. Klumpner TT, Lange EMS, Ahmed HS, Fitzgerald PC, Wong CA, Toledo P. An in vitro evaluation of the pressure generated during programmed intermittent epidural bolus injection at varying infusion delivery speeds. J Clin Anesth. 2016:34:632-7.

15. Dinges E, Heier J, Bollag L. It's not easy being green, or is it? Alkaline battery versus a/C power for programmed intermittent epidural bolus pumps. Int J Obstet Anesth Elsevier. 2018;33:100-1.

Ready to submit your research? Choose BMC and benefit from:

- fast, convenient online submission

- thorough peer review by experienced researchers in your field

- rapid publication on acceptance

- support for research data, including large and complex data types

- gold Open Access which fosters wider collaboration and increased citations

- maximum visibility for your research: over $100 \mathrm{M}$ website views per year

At $\mathrm{BMC}$, research is always in progress.

Learn more biomedcentral.com/submissions 